\title{
Convexity of sublevel sets of plurisubharmonic extremal functions
}

\author{
by Finnur Lárusson (London, Ont.), Patrice Lassere (Toulouse), \\ and Ragnar Sigurdsson (Reykjavík)
}

\begin{abstract}
Let $X$ be a convex domain in $\mathbb{C}^{n}$ and let $E$ be a convex subset of $X$. The relative extremal function $u_{E, X}$ for $E$ in $X$ is the supremum of the class of plurisubharmonic functions $v \leq 0$ on $X$ with $v \leq-1$ on $E$. We show that if $E$ is either open or compact, then the sublevel sets of $u_{E, X}$ are convex. The proof uses the theory of envelopes of disc functionals and a new result on Blaschke products.
\end{abstract}

1. Introduction. If $X$ is a complex manifold and $E$ is a subset of $X$, then the relative extremal function for $E$ in $X$ is defined as

$$
u_{E, X}=\sup \left\{v \in \operatorname{PSH}(X): v \leq-\chi_{E}\right\},
$$

where $\operatorname{PSH}(X)$ denotes the class of plurisubharmonic functions on $X$ and $\chi_{E}$ is the characteristic function of $E$, i.e., $\chi_{E}=1$ on $E$ and $\chi_{E}=0$ on $X \backslash E$. The main result of this paper is the following theorem.

Theorem 1. Let $X$ be a convex domain in $\mathbb{C}^{n}$ and let $E$ be a convex subset of $X$. If $E$ is either open or compact, then the sublevel sets

$$
\left\{\zeta \in X: u_{E, X}(\zeta)<\alpha\right\}
$$

of the relative extremal function for $E$ in $X$ are convex for all $\alpha \in[-1,0]$.

Convexity of sublevel sets of various extremal functions has been studied by several authors. If $X$ is a domain in $\mathbb{C}$ and $E \subset X$ is compact, then $u_{E, X}$ is harmonic in $X \backslash E$, and satisfies the boundary conditions $u_{E, X}=0$ on $\partial X$ and $u_{E, X}=-1$ on $\partial E$. The convexity of the level sets of the solution of the boundary value problem $\Delta u=0$ on $X \backslash E, u=0$ on $\partial X$, and $u=-1$ on

1991 Mathematics Subject Classification: Primary 32F05, 30D50; Secondary 31C10.

Key words and phrases: plurisubharmonic, relative extremal function, convex, disc functional, envelope, Blaschke product.

The first-named author was supported in part by the Natural Sciences and Engineering Research Council of Canada. 
$\partial E$, where $X$ is a bounded convex domain in $\mathbb{R}^{n}$ and $E$ is a compact convex subset of $X$, has been proved by several authors. See Papadimitrakis [1992], Rosay and Rudin [1989], and the references therein.

The pluricomplex Green function $G$ of a domain $X$ in $\mathbb{C}^{n}$ with a pole at $p$ in $X$ is the supremum of the class of negative plurisubharmonic functions $v$ on $X$ such that $v-\log |\cdot-p|$ is bounded above near $p$. If $X$ is bounded and convex, then the sublevel sets of $G$ are convex. See Momm [1994].

The pluricomplex Green function of a subset $E$ of $\mathbb{C}^{n}$ with a logarithmic pole at infinity is defined by the formula $V_{E}=\sup \{v \in \mathcal{L}: v \leq 0$ on $E\}$, where $\mathcal{L}$ denotes the set of plurisubharmonic functions $v$ on $\mathbb{C}^{n}$ such that $v-\log ^{+}|\cdot|$ is bounded above. Lempert has proved that the sublevel sets of $V_{E}$ are convex if $E$ is convex, compact, and non-pluripolar. See Momm [1996].

The proof of Theorem 1 is based on the theory of envelopes of disc functionals, which we will discuss in Section 2, and the following result on Blaschke products, which is of independent interest and will be proved in Section 3.

TheOrem 2. Let $S_{1}$ and $S_{2}$ be Borel subsets of the unit circle with $\lambda\left(S_{1}\right), \lambda\left(S_{2}\right)>\beta$. Then there are Blaschke products $\varphi_{1}$ and $\varphi_{2}$ preserving the origin with

$$
\lambda\left(\check{\varphi}_{1}^{-1}\left(S_{1}\right) \cap \check{\varphi}_{2}^{-1}\left(S_{2}\right)\right)>\beta .
$$

Here, $\lambda$ denotes the arc length measure on the unit circle $\mathbb{T}$. Also, for a bounded holomorphic function $\varphi$ on the unit disc $\mathbb{D}$ in $\mathbb{C}$, we denote by $\check{\varphi}$ the Borel measurable function on $\mathbb{T}$ whose value at almost every $x \in \mathbb{T}$ is the non-tangential limit of $\varphi$ at $x$.

Roughly speaking, Theorem 2 states that two Borel sets of equal length on $\mathbb{T}$ can almost be expressed as the images of a single Borel set on $\mathbb{T}$ by two Blaschke products preserving the origin.

The preprint of Edigarian and Poletsky [1997], which came to our attention after we completed this paper, essentially contains a proof of Theorem 2, very similar to ours.

After this paper was submitted for publication, we received a preprint from J. Duval, entitled Sur la fonction extrémale plurisousharmonique relative à deux convexes, containing a different proof of Theorem 1 .

2. Extremal functions as envelopes of disc functionals. Let $X$ be a domain in $\mathbb{C}^{n}$. An analytic disc in $X$ is a holomorphic map $f: \mathbb{D} \rightarrow X$. We say that $f$ is closed if $f$ can be extended to a holomorphic map on some neighbourhood of the closure $\overline{\mathbb{D}}$ of $\mathbb{D}$ with values in $X$, and we say that $f$ is bounded if $f(\mathbb{D})$ is relatively compact in $X$. We let $\mathcal{A}_{X}$ denote the set of 
all closed analytic discs in $X$ and $\mathcal{B}_{X}$ denote the set of all bounded analytic discs in $X$. Clearly, $\mathcal{A}_{X} \subset \mathcal{B}_{X}$.

If $f \in \mathcal{B}_{X}$, then $f$ has a non-tangential limit at almost every point in $\mathbb{T}$, and the limits define a Borel measurable function $\check{f}: \mathbb{T} \rightarrow X$. If $f \in \mathcal{A}_{X}$, then $\check{f}=g \mid \mathbb{T}$, where $g$ is a holomorphic extension of $f$ to some neighbourhood of $\overline{\mathbb{D}}$.

For every upper semicontinuous function $\varphi: X \rightarrow \mathbb{R} \cup\{-\infty\}$ we define the disc functional $H_{\varphi}: \mathcal{B}_{X} \rightarrow \mathbb{R} \cup\{-\infty\}$ by the formula

$$
H_{\varphi}(f)=\frac{1}{2 \pi} \int_{\mathbb{T}} \varphi \circ \check{f} d \lambda, \quad f \in \mathcal{B}_{X} .
$$

If $v \in \operatorname{PSH}(X), v \leq \varphi, f \in \mathcal{B}_{X}$, and $f(0)=\zeta$, then for $r \in(0,1)$,

$$
v(\zeta) \leq \frac{1}{2 \pi} \int_{0}^{2 \pi} v \circ f\left(r e^{i \theta}\right) d \theta \leq \frac{1}{2 \pi} \int_{\mathbb{T}} v \circ \check{f} d \lambda \leq H_{\varphi}(f) .
$$

The envelope of the functional $H_{\varphi}$ is the function $E H_{\varphi}: X \rightarrow \mathbb{R} \cup\{-\infty\}$ defined by the formula

$$
E H_{\varphi}(\zeta)=\inf \left\{H_{\varphi}(f): f \in \mathcal{A}_{X}, f(0)=\zeta\right\}, \quad \zeta \in X .
$$

The inequality (2.1) implies that $\sup \{v \in \operatorname{PSH}(X): v \leq \varphi\} \leq E H_{\varphi}$, and by taking for $f$ the constant $\zeta$, we see that $E H_{\varphi} \leq \varphi$. By a theorem of Poletsky [1991, 1993], $E H_{\varphi}$ is plurisubharmonic for every upper semicontinuous function $\varphi$, so we have

$$
\sup \{v \in \operatorname{PSH}(X): v \leq \varphi\}=E H_{\varphi} .
$$

See Lárusson and Sigurdsson [1996] for an alternative proof of Poletsky's theorem with generalizations to manifolds.

If $E$ is an open subset of $X$, then $\varphi=-\chi_{E}$ is upper semicontinuous and

$$
\int_{\mathbb{T}} \chi_{E} \circ \check{f} d \lambda=\lambda\left(\check{f}^{-1}(E)\right),
$$

so by (2.1) and Poletsky's theorem,

$$
\begin{aligned}
u_{E, X}(\zeta) & =-\frac{1}{2 \pi} \sup \left\{\lambda\left(\check{f}^{-1}(E)\right): f \in \mathcal{B}_{X}, f(0)=\zeta\right\} \\
& =-\frac{1}{2 \pi} \sup \left\{\lambda\left(\check{f}^{-1}(E)\right): f \in \mathcal{A}_{X}, f(0)=\zeta\right\}, \quad \zeta \in X .
\end{aligned}
$$

In particular, $u_{E, X}$ is plurisubharmonic on $X$. (When $E$ is not open, $u_{E, X}$ may fail to be upper semicontinuous.)

The proof of Theorem 1 is based on the formula (2.2) for $u_{E, X}$. Namely, assume that $X$ is a convex domain in $\mathbb{C}^{n}$ and $E$ is an open convex subset of $X$. Take two points $\zeta_{1}$ and $\zeta_{2}$ in $X$ such that $u_{E, X}\left(\zeta_{j}\right)<\alpha, j=1,2$. By 
(2.2), there are holomorphic maps $f_{j}: U \rightarrow X, j=1,2$, defined in some neighbourhood $U$ of $\overline{\mathbb{D}}$, such that $f_{j}(0)=\zeta_{j}$ and $\lambda\left(\check{f}_{j}^{-1}(E)\right)>-2 \pi \alpha$.

By Theorem 2, there are Blaschke products $\varphi_{j}: \mathbb{D} \rightarrow \mathbb{D}, j=1,2$, such that $\varphi_{j}(0)=0$ and $S=\check{\varphi}_{1}^{-1}\left(\check{f}_{1}^{-1}(E)\right) \cap \check{\varphi}_{2}^{-1}\left(\check{f}_{2}^{-1}(E)\right)$ has $\lambda(S)>-2 \pi \alpha$. Let $0<t<1$. Since $X$ is convex,

$$
f=t f_{1} \circ \varphi_{1}+(1-t) f_{2} \circ \varphi_{2}
$$

is a bounded analytic disc in $X$ with $f(0)=t \zeta_{1}+(1-t) \zeta_{2}$. Since $E$ is convex, $S \subset \check{f}^{-1}(E)$, so by $(2.2)$,

$$
u_{E, X}\left(t \zeta_{1}+(1-t) \zeta_{2}\right) \leq-\frac{1}{2 \pi} \lambda(S)<\alpha .
$$

This proves Theorem 1 in case $E$ is open. Assume now that $E$ is compact. Let $\left(V_{n}\right)$ be a decreasing basis of convex open neighbourhoods for $E$. Then $E=\bigcap \bar{V}_{n}$, so by Klimek [1991, Proposition 4.5.10], $u_{\bar{V}_{n}, X} \nearrow u_{E, X}$, and hence $u_{V_{n}, X} \nearrow u_{E, X}$. Therefore,

$$
u_{E, X}^{-1}[-1, \alpha)=\bigcup_{m=1}^{\infty} \bigcap_{n=1}^{\infty} u_{V_{n}, X}^{-1}[-1, \alpha-1 / m)
$$

is convex, and Theorem 1 is proved.

3. Boundary preimages under Blaschke products. Let us begin by observing that if $(a, b)$ is an interval on the real axis, then the bounded harmonic function $v$ in the upper half plane $\mathbb{H}$, which has boundary values equal to 1 on $(a, b)$ and 0 on $\mathbb{R} \backslash[a, b]$, is given at $z \in \mathbb{H}$ as the angle between $b-z$ and $a-z$ divided by $\pi$, i.e.,

$$
v(z)=\frac{1}{\pi} \operatorname{Arg} \frac{z-b}{z-a},
$$

where $\operatorname{Arg}: \mathbb{C} \backslash(-\infty, 0] \rightarrow(-\pi, \pi)$ is the principal branch of the argument.

Let $A \neq \mathbb{T}$ be the union of a finite number of non-empty open arcs $I_{j}, j=1, \ldots, m, m \geq 1$, in $\mathbb{T}$, whose closures are mutually disjoint, with endpoints $a_{j} \neq b_{j}$ listed counterclockwise. Let $\sigma: \mathbb{D} \rightarrow \mathbb{H}$ be a biholomorphic map which sends a point in $\mathbb{T} \backslash \bar{A}$ to $\infty$. Then $\sigma$ maps the $\operatorname{arcs} I_{j}$ onto bounded open intervals in $\mathbb{R}$. From the above formula for $v$ we see that the harmonic function $u_{A}$ in $\mathbb{D}$ having boundary values 1 on $A$ and 0 on $\mathbb{T} \backslash \bar{A}$ is given by the formula

$$
u_{A}(z)=\int_{\mathbb{T}} P(z, \cdot) \chi_{A} d \lambda=\frac{1}{\pi} \sum_{j=1}^{m} \operatorname{Arg} \frac{\sigma(z)-\sigma\left(b_{j}\right)}{\sigma(z)-\sigma\left(a_{j}\right)}, \quad z \in \mathbb{D},
$$

where $P$ denotes the Poisson kernel for $\mathbb{D}$. Observe that $u_{A}(0)=\lambda(A) /(2 \pi)$. 
If $z \in \mathbb{D}$, then the complex numbers

$$
\frac{\sigma(z)-\sigma\left(b_{j}\right)}{\sigma(z)-\sigma\left(a_{j}\right)}, \quad j=1, \ldots, m,
$$

are in $\mathbb{H}$ and the sum of their arguments is less than $\pi$. Hence their product is also in $\mathbb{H}$, and we can write $u_{A}=\operatorname{Re} F_{A}$, where

$$
F_{A}=\frac{1}{i \pi} \log \left(c_{A} \prod_{j=1}^{m} \frac{\sigma-\sigma\left(b_{j}\right)}{\sigma-\sigma\left(a_{j}\right)}\right) \in \mathcal{O}(\mathbb{D}) .
$$

Here, Log denotes the principal branch of the logarithm, and we take

$$
c_{A}=\left|\prod_{j=1}^{m} \frac{\sigma(0)-\sigma\left(a_{j}\right)}{\sigma(0)-\sigma\left(b_{j}\right)}\right|>0,
$$

so that $\operatorname{Im} F_{A}(0)=0$. Note that $F_{A}$ depends only on $A$, and not on $\sigma$.

The rational function

$$
B_{A}=\sigma^{-1} \circ\left(c_{A} \prod_{j=1}^{m} \frac{\sigma-\sigma\left(b_{j}\right)}{\sigma-\sigma\left(a_{j}\right)}\right)=\sigma^{-1} \circ e^{i \pi F_{A}}
$$

is a finite Blaschke product, for it maps $\mathbb{D}$ into $\mathbb{D}$ and $\mathbb{T}$ into $\mathbb{T}$. Observe that

$$
B_{A}(0)=\sigma^{-1}\left(e^{i \lambda(A) / 2}\right) \text {, }
$$

and

$$
A=\left(e^{i \pi F_{A}}\right)^{-1}(-\infty, 0)=B_{A}^{-1}(J)
$$

where $J$ is the $\operatorname{arc} \sigma^{-1}(-\infty, 0)$ in $\mathbb{T}$.

By Sard's theorem, there are automorphisms $\psi$ of $\mathbb{D}$ arbitrarily close to the identity such that $u_{A}(\psi(0))$ is a regular value for $u_{A}$. Then $u_{A}(\psi(0))$ is a regular value for $u_{A} \circ \psi=u_{\psi^{-1}(A)}$. This shows that for every $\varepsilon>0$, there is a finite union $A^{\prime}$ of open arcs in $\mathbb{T}$ as above with $\lambda\left(A \triangle A^{\prime}\right)<\varepsilon$, such that $u_{A^{\prime}}(0)$ is a regular value for $u_{A^{\prime}}$, and hence $B_{A^{\prime}}(0)$ is a regular value for $B_{A^{\prime}}$. Here, $\triangle$ denotes the symmetric difference.

Turning now to the proof of Theorem 2, let $S_{1}$ and $S_{2}$ be Borel sets in $\mathbb{T}$. Without loss of generality, we may assume that $\lambda\left(S_{1}\right)=\lambda\left(S_{2}\right)$. By regularity of the arc length measure, there are finite unions $A_{1}$ and $A_{2}$ of open $\operatorname{arcs}$ in $\mathbb{T}$ as above such that $\lambda\left(S_{j} \triangle A_{j}\right), j=1,2$, are arbitrarily small, and $\lambda\left(A_{1}\right)=\lambda\left(A_{2}\right)$. Let $B_{1}$ and $B_{2}$ be the finite Blaschke products associated with $A_{1}$ and $A_{2}$ as above. Since $\lambda\left(A_{1}\right)=\lambda\left(A_{2}\right)$, we have $B_{1}(0)=B_{2}(0)$. Let $C$ be the set of critical values of $B_{1}$ in $\mathbb{D}$, which is a finite subset of $\mathbb{D}$. By the above, we may assume that $B_{1}(0) \notin C$.

Let $\varphi_{2}: \mathbb{D} \rightarrow \mathbb{D} \backslash B_{2}^{-1}(C)$ be a universal covering map with $\varphi_{2}(0)=0$. We claim that $\varphi_{2}$ is a Blaschke product. It is then an infinite Blaschke product, unless $C=\emptyset$, which means that $A_{2}$ consists of a single arc. First of all, $\check{\varphi}_{2}^{-1}\left(B_{2}^{-1}(C)\right)$ is null, because $\check{\varphi}_{2}$ cannot be constant on a set of non-zero 
measure. Let us show that the non-tangential boundary values of $\varphi_{2}$ lie in the boundary of $\mathbb{D} \backslash B_{2}^{-1}(C)$. Generally, let $U$ be a bounded domain in $\mathbb{C}$ and $\varphi: \mathbb{D} \rightarrow U$ be the universal covering map. Suppose $x \in \mathbb{T}$ has $\check{\varphi}(x) \in U$. Let $V$ be a compact disc in $U$ containing $\check{\varphi}(x)$. Then there is a line segment $L=x(1-\varepsilon, 1), \varepsilon>0$, such that $\varphi(L) \subset V$ and $L \subset \varphi^{-1}(V)$. But $\varphi^{-1}(V)$ is a disjoint union of compact domains in $\mathbb{D}$, so it cannot contain $L$. Hence, all the non-tangential boundary values of $\varphi$ lie in the boundary of $U$. This shows that $\varphi_{2}$ is an inner function. Also, 0 is not a non-tangential boundary value of $\varphi_{2}$ because $0 \notin B_{2}^{-1}(C)$. Hence, $\varphi_{2}$ is a Blaschke product by Frostman's theorem. See Noshiro [1960, p. 33].

Now the composition $B_{2} \circ \varphi_{2}: \mathbb{D} \rightarrow \mathbb{D} \backslash C$ lifts by the covering map $B_{1}: \mathbb{D} \backslash B_{1}^{-1}(C) \rightarrow \mathbb{D} \backslash C$ to a holomorphic map $\varphi_{1}: \mathbb{D} \rightarrow \mathbb{D} \backslash B_{1}^{-1}(C)$ with $\varphi_{1}(0)=0$, as shown in the following diagram.

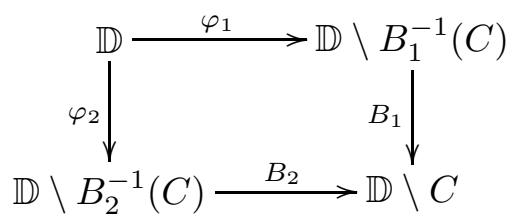

Since the non-tangential boundary values of $B_{2} \circ \varphi_{2}$ lie in $\mathbb{T} \cup C$, the nontangential boundary values of $\varphi_{1}$ lie in $\mathbb{T} \cup B_{1}^{-1}(C)$. Hence, $\varphi_{1}$ is inner, and 0 is not a non-tangential boundary value of $\varphi_{1}$, so by Frostman's theorem, $\varphi_{1}$ is a Blaschke product. Furthermore,

$$
\check{\varphi}_{1}^{-1}\left(A_{1}\right)=\check{\varphi}_{1}^{-1}\left(B_{1}^{-1}(J)\right)=\check{\varphi}_{2}^{-1}\left(B_{2}^{-1}(J)\right)=\check{\varphi}_{2}^{-1}\left(A_{2}\right) .
$$

To conclude the proof of Theorem 2, we shall show that if $\varphi$ is an inner function with $\varphi(0)=0$, and $S$ is a Borel set in $\mathbb{T}$, then $\lambda\left(\check{\varphi}^{-1}(S)\right)=\lambda(S)$. In other words, $\check{\varphi}_{*} \lambda=\lambda$. For an $L^{\infty}$ function $g$ on $\mathbb{T}$, we let $H[g]$ denote the bounded harmonic function on $\mathbb{D}$ with non-tangential limit $g(x)$ at almost every $x \in \mathbb{T}$.

First we consider the case when $S$ is an arc. Suppose $\varphi$ has a nontangential limit in $\mathbb{T}$ at $x \in \mathbb{T}$, and let $z_{n} \rightarrow x$ non-tangentially in $\mathbb{D}$. Then $\varphi\left(z_{n}\right) \rightarrow \check{\varphi}(x) \in \mathbb{T}$. If $\check{\varphi}(x)$ is not an endpoint of $S$, then $\chi_{S}$ is continuous at $\check{\varphi}(x)$, so

$$
\left(H\left[\chi_{S}\right] \circ \varphi\right)\left(z_{n}\right) \rightarrow \chi_{S}(\check{\varphi}(x))=\chi_{\check{\varphi}^{-1}(S)}(x) .
$$

Now $\check{\varphi}$ cannot be constant on a set of non-zero measure, so the preimage under $\check{\varphi}$ of the endpoints of $S$ is a nullset. Therefore, the bounded harmonic function $H\left[\chi_{S}\right] \circ \varphi$ has non-tangential limit $\chi_{\check{\varphi}^{-1}(S)}(x)$ at almost every $x \in \mathbb{T}$, so $H\left[\chi_{S}\right] \circ \varphi=H\left[\chi_{\check{\varphi}^{-1}(S)}\right]$. Hence,

$$
\frac{\lambda(S)}{2 \pi}=H\left[\chi_{S}\right](0)=\left(H\left[\chi_{S}\right] \circ \varphi\right)(0)=H\left[\chi_{\check{\varphi}^{-1}(S)}\right](0)=\frac{\lambda\left(\check{\varphi}^{-1}(S)\right)}{2 \pi} .
$$


This shows that $\lambda\left(\check{\varphi}^{-1}(S)\right)=\lambda(S)$ for open sets and hence for compact sets. In the general case,

$$
\begin{aligned}
\lambda(S) & =\sup \lambda(K)=\sup \lambda\left(\check{\varphi}^{-1}(K)\right) \\
& \leq \lambda\left(\check{\varphi}^{-1}(S)\right) \leq \inf \lambda\left(\check{\varphi}^{-1}(U)\right)=\inf \lambda(U)=\lambda(S),
\end{aligned}
$$

where the supremum is taken over compact subsets $K$ of $S$, and the infimum is taken over open supersets $U$ of $S$.

\section{References}

A. Edigarian and E. A. Poletsky, Product property of the relative extremal function, preprint, 1997.

M. Klimek, Pluripotential Theory, Oxford Univ. Press, 1991.

F. Lárusson and R. Sigurdsson, Plurisubharmonic functions and analytic discs on manifolds, Report RH-15-96, Science Institute, University of Iceland, 1996.

S. Momm, Boundary behavior of extremal plurisubharmonic functions, Acta Math. 172 (1994), 51-75.

-, An extremal plurisubharmonic function associated to a convex pluricomplex Green function with pole at infinity, J. Reine Angew. Math. 471 (1996), 139-163.

K. Noshiro, Cluster Sets, Ergeb. Math. Grenzgeb. 28, Springer, 1960.

M. Papadimitrakis, On convexity of level curves of harmonic functions in the hyperbolic plane, Proc. Amer. Math. Soc. 114 (1992), 695-698.

E. A. Poletsky, Plurisubharmonic functions as solutions of variational problems, in: Proc. Sympos. Pure Math. 52, Part 1, Amer. Math. Soc., 1991, 163-171.

—, Holomorphic currents, Indiana Univ. Math. J. 42 (1993), 85-144.

J.-P. Rosay and W. Rudin, A maximum principle for sums of subharmonic functions, and the convexity of level sets, Michigan Math. J. 36 (1989), 95-111.

Department of Mathematics

University of Western Ontario

London, Ontario N6A 5B7

Canada

E-mail: larusson@uwo.ca

Laboratoire de Mathématiques E. Picard

Université Paul Sabatier-Toulouse 3

118 route de Narbonne

F-31062 Toulouse Cedex, France

E-mail: lassere@picard.ups-tlse.fr
Science Institute University of Iceland Dunhaga 3 IS-107 Reykjavík, Iceland E-mail: ragnar@raunvis.hi.is 